Richard G. Gibson, Department of Mathematics, Columbus State University, Columbus, Georgia, 31907, USA, email: Gibson_Richard@@colstate.edu

\title{
CONCERNING A CHARACTERIZATION OF CONTINUITY
}

B. D. Smith in [8] gave a characterization of continuity for real-valued functions defined on $[0,1]$ by proving that a function $f:[0,1] \mapsto \mathbb{R}$ is continuous if and only if

(1) $f$ is almost continuous in the sense of Stallings, [9].

(2) $f$ is almost continuous in the sense of Husain, [5], and

(3) $f$ is not of the Cesaro type.

The examples given by Smith in [8] and by R. J. Fleissner in [3] show that the three conditions (1), (2) and (3) are not redundant.

In reference to the paper of Smith, Jack Brown in [1] proved that the Cesaro-Vietoris function is almost continuous in the sense of Stallings. Using the Cesaro-Vietoris function and the examples of Smith one can also show that the three conditions are not redundant.

In what follows, two problems related to this characterization of continuity will be discussed.

In the first problem " $f$ is almost continuous in the sense of Stallings" will be replaced with a weaker condition " $f$ is a Darboux function" and it will be shown that the characterization of continuity remains true. Also it follows that for the classes of functions considered, " $f$ is a Darboux function" is the weakest possible condition for which the characterization remains true.

In the second problem " $f$ is almost continuous in the sense of Stallings" will be replaced with a stronger condition " $f$ is an extendable function". Then it will be shown that this condition and conditions (2) and (3) are not redundant.

Let $X$ and $Y$ be topological spaces and let $f: X \mapsto Y$ be a function. Then:

D: $f$ is a Darboux function, if $f(C)$ is a connected subset of $Y$ whenever $C$ is a connected subset of $X$.

Key Words: extendable function, connectivity function, almost continuity

Mathematical Reviews subject classification: 26A15

Received by the editors October 21, 1991 
Conn: $f$ is a connectivity function, if the graph of $f$ restricted to $C$ is connected in $X \times Y$ whenever $C$ is a connected subset of $X$.

ACS: $f$ is an almost continuous function in the sense of Stallings if and only if for any open subset $U$ of $X \times Y$ containing the graph of $f, U$ contains the graph of a continuous function $g: X \mapsto Y$.

Ext: $f$ is an extendable function if and only if there exists a connectivity function $g: X \times[0,1] \mapsto Y$ such that $f(x)=g((x, 0))$ for each $x \in X$.

PC: $f$ is peripherally continuous, if for each $x \in X$ and for each pair of open sets $U$ and $V$ containing $x$ and $f(x)$, respectively, there exists an open subset $W$ of $U$ containing $x$ such that $f(b d(W))$ is a subset of $V$, where $b d(W)$ denotes the boundary of $W$.

For real-valued functions $f:[0,1] \mapsto \mathbb{R}$ we have only the following implications among the classes of functions defined above. See the paper by Brown, Humke and Laczkovich, [2].

$$
E x t \longrightarrow A C S \longrightarrow C o n n \longrightarrow D \longrightarrow P C
$$

ACH: $f$ is an almost continuous function in the sense of Husain if and only if for each $x \in X$ and for each open set $V$ containing $f(x), c l\left(f^{-1}(V)\right)$ is a neighborhood of $x$, where $c l$ denotes the closure.

CT: $f$ is of the Cesaro type if and only if there exist nonempty open subsets $U$ of $X$ and $V$ of $Y$ such that for each $y \in V, f^{-1}(y)$ is dense in $U$.

Cliq: Let $(Y, d)$ be a metric space. $f$ is said to be cliquish if and only if for each $\epsilon>0$, every nonempty open subset $U$ of $X$ contains a nonempty open subset $W$ such that $d(f(x), f(y))<\epsilon$ whenever $x, y \in W$.

To extend the characterization of continuity given by B. D. Smith in [8], J. Smital and E. Stanova in [7] required that the domain of the function be a $T_{3}$ locally connected Baire topological space. Their theorem is as follows.

Theorem. Let $X$ be a $T_{3}$ locally connected Baire topological space. A function $f: X \mapsto \mathbb{R}$ is continuous if and only if

(1) $f$ is almost continuous in the sense of Stallings,

(2) $f$ is almost continuous in the sense of Husain, and

(3) $f$ is not of Cesaro type. 
The first result given in this paper is Theorem 1 which is similar to the above theorem of Smital and Stanova. However in Theorem 1 " $f$ is almost continuous in the sense of Stallings" is replaced with " $f$ is a Darboux function".

Theorem 1. Let $X$ be a $T_{3}$ locally connected Baire topological space. A function $f: X \mapsto \mathbb{R}$ is continuous if and only if

(1) $f$ is a Darboux function,

(2) $f$ is almost continuous in the sense of Husain, and

(3) $f$ is not of Cesaro type.

The only new ingredient in the course of the proof of Theorem 1 is the following easy observation, the proof of which we leave to the reader.

Lemma 1. Let $X$ be a $T_{1}$ topological space and let $f: X \mapsto \mathbb{R}$ be a Darboux function. Assume that there is a closed connected subset $F$ of $X$ and some $y \in \mathbb{R}$ such that $y$ is not in $f(F)$. Then $y$ is an upper-bound or a lower-bound of $f(F)$.

Having Lemma 1, the method of proof given in [7] can be used to obtain the following.

Lemma 2. Let $X$ be a locally connected $T_{3}$ Baire topological space. Let $f$ : $X \mapsto \mathbb{R}$ be a Darboux function not of Cesaro type. Then each nonempty open set $U$ contains a nonempty open set $W$ such that $f$ is bounded on $W$.

Lemma 3. Let $X$ be a $T_{3}$ locally connected topological space. Let $f: X \mapsto \mathbb{R}$ be a Darboux function not of Cesaro type. Let $U$ be a nonempty open set and let $\delta>0$ such that $|f(x)-f(y)|<\delta$ for $x, y \in U$. Then there is a nonempty open subset $W$ of $U$ such that $|f(x)-f(y)|<(2 / 3) \delta$ for $x, y \in W$.

Theorem 2. Let $X$ be a $T_{3}$ locally connected Baire topological space. If $f$ : $X \mapsto \mathbb{R}$ is a Darboux function not of Cesaro type, then $f$ is cliquish.

Theorem 3. Let $X$ be a topological space, let $(Y, d)$ be a metric space, and let $f: X \mapsto Y$ be a function. Then $f$ is continuous if and only if $f$ is both cliquish and almost continuous in the sense of Husain.

From the above lemmas and theorems the proof of Theorem 1 is now complete. We now show that among the classes of functions Ext, ACS, Conn, $D$ and $P C$, " $f$ is a Darboux function" is the weakest condition for which the characterization of continuity is true. 
Theorem 4. If $f:[0,1] \mapsto \mathbb{R}$ is an almost continuous function in the sense of Husain, then $f$ is peripherally continuous.

Proof. Assume that $f:[0,1] \mapsto \mathbb{R}$ is an almost continuous function in the sense of Husain. To show that $f$ is peripherally continuous we use a result of J. Young [10]. Thus we need only to prove that for each $x \in[0,1]$ there exist sequences $x_{n} \nearrow x$ and $y_{n} \searrow x$ such that $f\left(x_{n}\right) \rightarrow f(x)$ and $f\left(y_{n}\right) \rightarrow f(x)$.

Select any $x \in[0,1]$ that is not an endpoint and let $\epsilon_{n}>0$, where $n$ is a positive integer. Let $V_{n}$ be the open interval $\left(f(x)-\epsilon_{n}, f(x)+\epsilon_{n}\right)$. Since $f$ is almost continuous in the sense of Husain, $c l\left(f^{-1}\left(V_{n}\right)\right)$ is a neighborhood of $x$. Now there exist $a_{n}, b_{n} \in \operatorname{cl}\left(f^{-1}\left(V_{n}\right)\right)$ such that $x-\epsilon_{n}<a_{n}<x<b_{n}<x+\epsilon_{n}$.

If $a_{n}, b_{n}$ are not in $f^{-1}\left(V_{n}\right)$, then there exist $x_{n}, y_{n} \in f^{-1}\left(V_{n}\right)$ such that $a_{n}<x_{n}<x<y_{n}<b_{n}$. Then $f\left(x_{n}\right), f\left(y_{n}\right) \in V_{n}$. If $a_{n}, b_{n}$ are in $f^{-1}\left(V_{n}\right)$, then let $x_{n}=a_{n}$ and $y_{n}=b_{n}$. In either case $f\left(x_{n}\right), f\left(y_{n}\right) \in V_{n}$.

Let $\epsilon_{n}>0$ be a sequence such that $\epsilon_{n} \rightarrow 0$. Thus there exist monotonic sequences $x_{n} \nearrow x$ and $y_{n} \searrow x$ such that $f\left(x_{n}\right) \rightarrow f(x)$ and $f\left(y_{n}\right) \rightarrow f(x)$.

If $x$ is an endpoint, then we have only a unilateral condition.

R. G. Gibson and F. Roush in [4] proved that there exist almost continuous functions in the sense of Stallings that are not extendable. The second result given in this paper shows that the following three conditions are not redundant.

(1) $f$ is extendable,

(2) $f$ is almost continuous in the sense of Husain, and

(3) $f$ is not of the Cesaro type.

Example 1. Let $f:[0,1] \mapsto[-1,1]$ be defined by

$$
f(x)= \begin{cases}x & \text { if } x \text { is rational } \\ -x & \text { if } x \text { is irrational }\end{cases}
$$

Now conditions (2) and (3) are true but condition (1) is not true.

Example 2. Let $f:[0,1] \mapsto[-1,1]$ be defined by

$$
f(x)= \begin{cases}\sin \frac{1}{x} & \text { if } x>0 \\ 0 & \text { if } x=0\end{cases}
$$

In this case (1) and (3) are true but condition (2) is not true. 
Example 3. We now give an example of a function that satisfies conditions (1) and (2) but does not satisfy condition (3). Rosen, Gibson and Roush in [6] constructed an extendable function $h:[0,1] \mapsto[0,1]$ which is dense in $[0,1] \times[0,1]$. Since each Darboux function which has a dense graph assumes every value in $(0,1)$ in every interval in $[0,1], h$ is almost continuous in the sense of Husain and is of the Cesaro type.

For the following question, we first make the following definition and observation.

Let $X$ and $Y$ be topological spaces and let $f: X \mapsto Y$ be a function. Then $f$ is quasicontinuous if for each $x \in X$ and for any pair of open sets $U$ and $V$ containing $x$ and $f(x)$, respectively, there exists a nonempty open subset $W$ of $U$ such that $f(W)$ is a subset of $V$.

Clearly each quasicontinuous function with range a subset of a metric space is cliquish.

Question. Does there exist an extendable function $f:[0,1] \mapsto \mathbb{R}$ not of the Cesaro type which is not quasicontinuous? In [7] Smital and Stanova constructed an almost continuous function in the sense of Stallings not of the Cesaro type which is not quasicontinuous.

\section{References}

[1] J. B. Brown, Almost continuity of the Cesaro-Vietoris function, Proc. Amer. Math. Soc. 49 (1975), 185-188.

[2] J. B. Brown, P. Humke, and M. Laczkovich, Measurable Darboux functions, Proc. Amer. Math. Soc. 102 (1988), no. 3, 603-609.

[3] R. J. Fleissner, An almost continuous function, Proc. Amer. Math. Soc. 45 (1974), 346-348.

[4] R. G. Gibson and F. Roush, Connectivity functions with a perfect road, Real Anal. Exchange 11 (1985-86), no. 1, 260-264.

[5] T. Husain, Almost continuous mappings, Prace Math. 10 (1966), 1-7.

[6] H. Rosen, R. G. Gibson, and F. Roush, Extendable functions and almost continuous functions with a perfect road, Real Anal. Exchange 17 (199192), no. 1, 248-257.

[7] J. Smital and E. Stanova, On almost continuous functions, Acta Math. Univ. Comen. 37 (1980), 147-155. 
[8] B. D. Smith, An alternate characterization of continuity, Proc. Amer. Math. Soc. 39 (1973), 318-320.

[9] J. Stallings, Fixed point theorems for connectivity maps, Fund. Math. 47 (1959), 249-263.

[10] J. Young, A theorem in the theory of functions of a real variable, Rend. Circ. Mat. Palermo 24 (1907), 187-192. 